\title{
Les ethnonymes : des ethnostylèmes révélateurs? Jalons pour une glossairistique littéraire comparée
}

\author{
Diane Schwob \\ ESPE Centre Val-de-Loire \\ Université d'Orléans \\ LLL-GORDF
}

Cette proposition de communication se situe dans la session du colloque consacrée à la francophonie. Nous y poserons la question des interactions entre la lexicographie différentielle interprétative d'une part, et la sémiotique littéraire des textes francophones d'autre part. Notre réflexion se fondera sur des analyses de corpus visant à chercher dans quelle mesure les ethnonymes, termes référant généralement à "des groupes linguistiques et culturels non constitués en entité étatique » (Dubois et al. [1973] 1994 : 186), seraient des ethnostylèmes révélateurs d'une écriture hétérolingue.

Parmi les ethnonymes, nous nous centrerons sur les emprunts lexématiques (Poirier 1995 : 32) qui, issus des langues locales, restent en quête d'adaptation phonique, phonologique et graphique (Queffélec 1998 : 246-248). Comme tout nom propre, les ethnonymes peuvent être soumis au processus d'emprunt (Guilbert 1975 : 92). Celui-ci, au tout début du processus, est perçu comme xénisme ; en s'installant plus durablement dans la langue-cible, il atteint le stade du pérégrinisme (Dubois et al., id. : 512). Nous considérons les emprunts lexématiques, en ce qu'ils sont «immédiatement repérables par un lecteur hexagonal » (Caitucoli $2007: 58$ ), comme une porte d'entrée visible sur une analyse ethnostylistique de la variation dans des textes hétérolingues, qui peut déboucher à son tour sur d'autres analyses - de l'emprunt sémantique, de phénomènes syntaxiques... - ainsi que sur des applications didactiques (Schwob 2014a).

Dans les trois romans analysés ici, le francais de référence (Poirier 1995 : 26) se mêle aux langues d'adstrat : au wolof dans Le Jujubier du patriarche ([1993] 1998) de Sow Fall, sénégalaise ; au malinké et au bambara, dans Allah n'est pas obligé (2000) de Kourouma, ivoirien ; à l'arabe dialectal libanais dans Le Rocher de Tanios ([1993] 2004), de Maalouf, franco-libanais. En effet, dans le cadre d'une norme pluricentrique (Poll 2005 : 17-28), les écrivains hétérolingues textualisent dans leurs œuvres le contact des langues, «idiomes étrangers [ou] variétés (sociales, régionales, chronologiques) de la langue auctoriale» (Grutman 1996 : 72). Cette textualisation manifeste à la fois une «appropriationpossession », par laquelle des locuteurs endogènes font du français leur propriété, et une « appropriationadaptation » par laquelle ils le rendent propre à exprimer une pensée endogène (Caitucoli 2007 : 54).

Pour mieux comprendre ces phénomènes d'appropriation, nous éclairerons le traitement des ethnonymes par les auteurs en nous penchant sur les emprunts lexématiques désignant des représentants des pouvoirs religieux et temporel. L'ensemble du corpus lexical ainsi délimité relève donc du champ de l'environnement politique au sens large. En effet, « tout ce qui peut exister autour de soi constitue, pour je (qui, lui seul, est un "non-lieu"), l'environnement et est susceptible de recevoir une dénomination », « et les relations mutuelles entre les hommes, leur nature politique, la circonscription des pouvoirs, [...] supposent ces systèmes de dénominations réciproques : eux et nous ». En francophonie spécialement, la relation entre l'homme et son environnement est manifeste, «l'espace nommé y jouant le rôle fondateur » (Cheriguen 1994 : 94-96).

Qu'est-ce qui ferait de ces ethnonymes balisant l'environnement politique, des ethnostylèmes révélateurs ? Si Mendo Zé, fondateur de l'ethnostylistique, a déjà montré la pertinence de l'étude des éléments onomastiques qui contribuent à poser l'univers référentiel du roman, par cette question, nous entendons chercher si l'ethnostylistique de l'emprunt (Ngo Lend 2010 : 147) peut en outre être considérée comme une entrée privilégiée dans une poétique romanesque interculturelle. Le style, c'est pour Molinié (1994: 204-205) le « regroupement déterminé de stylèmes », ceux-ci étant des corrélations reliant au moins deux éléments langagiers de nature quelconque. Pour Mendo Zé, les ethnostylèmes sont alors des «indices référentiels ou déictiques [...] permettant de situer le texte par rapport à la culture, à la langue et à la société occurrente, celle des lieux-sources textuels» (2009:17-18), des « unités linguistiques porteuses 
d'un archi-trait sémantique spécifique » (2010:37).

Toutefois l'entreprise ethnostylistique pose une question qui n'est pas sans importance : si l'ethnostylème suppose par définition la corrélation de deux éléments, et si l'emprunt est un de ces éléments, avec quoi l'ethnostylistique le mettra-t-il en relation? Sera-ce, en tant que signe, avec son référent, comme le laisse supposer la définition de l'ethnostylistique comme prise en compte de l'univers référentiel et culturel, des lieux sources de l'œuvre ? Et si ce n'est pas le cas, en quoi consistera son corrolaire ?

D'ailleurs, les analyses pragmatiques des littératures francophones ont déjà mis en lumière le rôle essentiel du contexte de l'œuvre littéraire (Maingueneau 1993), ce qui amène une deuxième question : estil alors besoin d'une ethnostylistique de l'emprunt, et pourquoi placer le point de départ des réflexions sur la littérature francophone en-deçà des analyses de discours, au niveau de la sémantique, voire de la sémiotique lexicale?

Pour en savoir plus, nous nous pencherons donc sur les fonctions remplies par ces ethnonymes dans la création d'un univers de référence littéraire, telles qu'elles se dévoilent notamment à partir d'une analyse de leur traitement, perçu comme le positionnement des auteurs vis-à-vis des contraintes que ces mots, à un niveau sémiotique, imposent sur leurs énoncés. Par traitement, nous entendons aussi bien la mise en scène graphique voire orthographique des lexies elles-mêmes, que la gestion de leurs récurrences et de leur cotexte glossairistique. À partir de là, nous chercherons quelle méthode d'analyse structurale du traitement des ethnonymes permettrait de mettre en regard des traits distinctifs pertinents dans le cadre d'un système suffisamment oppositif pour ouvrir la voie à une comparaison des stratégies scripturales mises en œuvre autour de ces termes par les auteurs du corpus, afin de poser quelques éléments de réponse à une troisième et dernière question : quels outils pour une ethnostylistique comparée ?

\section{L'ethnonyme : un signifiant flottant à la croisée des fonctions}

Tentons d'abord de définir l'objet de nos analyses. Le nom propre a pour fonction «d'identifier un référent, là où le lexème [...] sert au contraire à le catégoriser conceptuellement» (Gouvert 2008 : 147). Il est en général défini comme désignateur direct et rigide, "en vertu d'une convention ad hoc de dénomination qui associe directement et avec un lien durable la forme phonique ou graphique du [nom propre] au particulier visé » (Jonasson $1994: 65$ ). À ce titre, il peut représenter "l'idéal de noms purement référentiels ", dénués de signifiés (Rastier $1990: 32$ ), thèse battue en brèche notamment par Rastier (1990) ou Vaxelaire (2005). Sa réputation d'uniréférentialité en ferait aussi un «pur index, [...] pointé pour l'éternité et dans tous les mondes sur une et une seule personne » (Rastier 1990 : 33).

Anthroponymes collectifs, les ethnonymes entrent dans la classification des noms propres fondée sur leur référent extralinguistique établie par Bauer ([1985] 1998 : 53-59). Celui-ci relativise leur uniréférentialité : si le déterminant défini pluriel renvoie au peuple perçu comme collectivité - donc comme unité -, en revanche, le déterminant indéfini comme dans un / des Français, imprime au terme le fonctionnement d'un nom commun. S'ils ne sont pas forcément uniréférentiels, sont-ils toutefois des désignateurs rigides ? Amselle les voit plutôt comme des "signifiants flottants », pouvant recevoir « une multitude de sens en fonction des époques, des lieux ou des situations sociales » $(1985: 37-38)$ - l'ethnie, définie dans une dialectique entre "nous» et "eux», étant elle-même "un composé spécifique, en équilibre plus ou moins instable, de culturel et de social» (ibid. : 17), dont les frontières seront considérées « comme des barrières sémantiques ou des systèmes de classement » (ibid. : 34). Critiquant la notion d'« ethnie-substance », Amselle estime que « l'ethnologie et le colonialisme, [...] pressés de classer et de nommer », sont responsables d'un figement des «étiquettes ethniques» (ibid. : 10). L'ethnonyme a au fond une valeur performative : on peut tout à fait « se revendiquer comme Peul ou Bambara » quoiqu'il soit contestable de faire de ce mode d'identification « une essence » (ibid. : 37-38).

La question du référent est donc déterminante pour ces termes ; nous avons d'ailleurs sélectionné les lexies du corpus en fonction de leurs référents : peuples, mais aussi représentants du pouvoir. En lexicographie différentielle, le classement par le référent (Poirier 1995) oppose notamment les termes mettant en évidence une lacune linguistique liée à l'originalité du référent, à ceux qui ont un équivalent paradigmatique dans la langue emprunteuse, et dont l'emploi génére l'existence de doublets 
intersystémiques - relevant donc de deux systèmes linguistiques différents, par opposition aux doublets intrasystémiques (Benzakour $2000: 314)^{1}$. Les termes dénotant un référent original sont notamment les statalismes, renvoyant au sens strict aux spécificités administratives et politiques d'un pays (Pohl 1985).

Cette opposition pourrait se réinterpréter en termes de fonctions de l'emprunt lexématique. On peut en effet considérer que les termes à référent original remplissent une fonction dominante " cognitive » visant à l'identification du référent, tandis que les doublets pourraient remplir en discours des fonctions variées, et non exclusives, telles que les fonctions «sociale » et «expressive-créative » (Thibault \& Gle $\beta$ gen 2005 : III). Or, l'évaluation de la fonction dominante du doublet se fait souvent à partir du paradigme de la langue emprunteuse, alors que cet emploi, qui renvoie à des pratiques où bilingues et monolingues jouent des rôles spécifiques (Bitjaa Kody 2000), gagne à être évoqué aussi du point de vue de ces locuteurs bilingues, experts dans la langue prêteuse.

Benzakour souligne certes les motivations sociales présidant à l'emploi de ces emprunts, dont les valeurs diatopique et énonciative évoquent l'appartenance culturelle du locuteur (Benzakour 2000 : 320), ainsi que leur valeur ludique, qui les renvoie à la fonction expressive-créative. Mais elle s'interroge aussi sur l'existence de motivations internes, liées à la signification du doublet et à son fonctionnement référentiel, qui engageraient donc partiellement la fonction cognitive : pour elle, «c'est de l'intérieur d'abord que le doublet par emprunt lexical tire profondément sa raison d'être » (ibid. : 318). En effet, si l'emploi de termes à référent original renvoie à une valeur plutôt dénotative, permettant le repérage du référent (Kerbrat-Orecchioni 1977), en revanche, le doublet a «le même sens dénotatif que le vocable français avec lequel il coexiste, mais il présente en plus un contenu de connotation [...] latent, suggéré » (Benzakour 2000 : 319). Benzakour souligne notamment ses connotations comme valeurs associées, générant une «image intérieure faite de références socioculturelles puisées dans la source [endogène], nourrie de souvenirs, d'impressions sensibles et d'actions externes et internes du vécu socioculturel [endogène] ou de l'imaginaire populaire », bref, « tout un ensemble de valeurs que le sujet parlant associe au terme emprunté » (Benzakour 2000 : 321). Elle juge donc le doublet, « du point de vue cognitif, plus chargé que le vocable français cantonné à la fonction dénotative » (ibid. : 321).

Ainsi, la fonction des doublets par emprunt, loin d'être purement sociale, est aussi de traduire une certaine vision du monde, conformément à l'hypothèse sémantactique, selon laquelle le langage, "par-delà les contraintes "universelles" [...], n'est que la mise en forme d'une expérience perçue au travers du prisme culturel » (Manessy 1995 : 234). Or, Manessy décrit ainsi les rapports entre les fonctions cognitive et sociale dans le processus d'appropriation propre à la créolisation : " complexification et expansion n'ont rien à faire avec la fonction référentielle. Elles ne se développent pas pour que les gens se comprennent mieux, mais parce qu'ils se comprennent mieux ». En effet, «pour les membres d'une communauté, les situations de la vie courante ne comportent qu'un nombre limité de significations possibles », si bien que « la fonction référentielle ne s'exerce pas pour eux [...] dans les mêmes conditions qu'entre des interlocuteurs étrangers ; elle procède par allusions partiellement implicites à un système de signifiés communs» (1995:36). Il se pourrait alors que les ethnonymes empruntés, signes d'appropriation du français, soient tout spécialement situés à la croisée des fonctions cognitive et sociale, les auteurs négociant leur valeur référentielle entre rigidité et flottement.

\section{La sémantique interprétative au service de l'ethnostylistique}

Mendo Zé (2010 : 12-17) fonde l'ethnostylistique en réaction contre les écoles stylistiques pratiquant à ses yeux un «immanentisme radical», dans lequel « les structures linguistiques se définissent uniquement par les relations qui les unissent sans préoccupation extralinguistique » : ces écoles posent comme préablable à toute analyse stylistique l'autonomie du discours littéraire qui, puisqu'il fait apparaître " l'idée du référent dans son propre déroulement », devient à lui-même "son propre référent » (Mendo Zé, Tonye \& Noumssi 2009 : 8-9, citant Molinié \& Viala). Pour Mendo Zé, l'appartenance du texte littéraire à l'univers du discours implique nécessairement de prendre en compte la situation de communication, et avec elle, « les contraintes stylistico-thématiques affectant les considérations non linguistiques », qui sont d'ordres divers, «psychologique ou psychanalytique, ou d'ordre culturel ou encyclopédique », autrement dit, «l'ensemble des savoirs implicites sur le monde et l'ensemble des systèmes d'interprétation et 
d'évaluation de l'univers référentiel » (Mendo Zé 2010 : 21-22).

Ainsi envisagée, l'œuvre littéraire, puisqu'elle «s'inscrit dans un mouvement énonciatif à l'intérieur d'un discours parallèle au procédé narratif», peut être définie comme «le lieu de représentation de la parole et de la pensée non seulement de l'auteur mais d'un corps social plus complexe dont il actualise et modalise les tensions », si bien qu'elle s'identifie à « l'espace de la mémoire collective » (Barry 2006 : 14). Cette inscription se fait par les voies de la transposition culturelle, procédé par lequel l'auteur « introduit dans ses textes l'univers de croyance des personnages qu'il met en scène », « univers doxique qui requiert, pour être décodé, une connaissance de l'univers mis en scène » (Ngo Lend \& Fonkua 2010 : 133).

On le comprend, l'ethnostylistique fait donc de la «connexion entre la référence et le référent », le lieu même « de lisibilité du signe s'articulant sur l'idéologique ou sur un usage socio-culturel » (Mendo Zé, Tonye \& Noumssi 2009 : 12). Pour interpréter le texte, elle devra alors le rapporter en tant qu'énoncé à ses «lieux sources» et «lieux cibles» (Mendo Zé 2010 : 14). En effet, rendre compte de l'idéologicoculturel implique de s'inscrire dans le cadre d'une sémantique contextualiste, considérant comme unité du signe, non plus seulement le mot, «mais le texte, le discours où le sens n'est plus dans la relation de substitution paradigmatique ; mais dans la connexion syntagmatique qui génère un signifié particulier ou occasionnel ». Par conséquent, l'ethnostylistique se centrera sur « cet ordre de signifiance qu'engendre le discours, c'est-à-dire du sens construit, contenu du système connotatif », ce qui l'amène à dépasser "la fonction dénotative pour une valorisation du langage de connotations » (Mendo Zé 2010 : 24-25).

Nous trouvons dans la sémantique interprétative de Rastier, complétée par la sémantique des points de vue de Raccah, à la fois les fondements épistémologiques de l'ethnostylistique de Mendo Zé, et les linéaments d'une méthode d'analyse ethnostylistique compatible avec un immanentisme ouvert. En effet, chez Mendo Zé, le lien au référent, perdu avec les stylistiques prônant un immanentisme radical, se rétablit par la prise en compte du contexte endogène de la communication. Or, la sémantique interprétative de Rastier, postulant que « le sens n'est ni interne ni externe, mais immanent aux pratiques d'énonciation et d'interprétation ", replace le texte, contexte du signe, dans le contexte plus large de la situation de communication, ce qui fait de l'interprétation une pratique située, sociale et culturelle. Dans ce cadre, «un mot même est un passage : son expression est un extrait d'un texte : son contenu, un fragment d'un mythe » (Rastier, $2003: 36$ ).

C'est là que l'«immanentisme ouvert» (Mendo Zé 2010 : 27) espéré par Mendo Zé laisse imaginer la possibilité d'une ethnostylistique structuraliste propre à l'analyse méthodique des procédés de transposition culturelle. En effet, la sémantique interprétative, par analyse différentielle, décrit la production du sens comme un "parcours interprétatif» (Rastier 2007 : 10) défini en termes structuralistes, car « un mot ne peut être défini isolément, par rapport à ce qu'il désigne. Il doit l'être relativement à d'autres mots » (Rastier [1991] 2001 : 104). C'est en mettant l'accent sur les connotés produits en cotexte et dans le contexte de la situation d'énonciation, en considérant le langage comme « un système différentiel d'oppositions », et non un « codage d'identités référentielles » (Rastier 2003 : 26), que la sémantique interprétative, à la fois fonde théoriquement l'ethnostylistique, et lui offre des méthodes d'analyse compatibles avec un structuralisme garant de scientificité. De fait, si l'ethnostylème mettait en relation le signe avec son référent, l'entreprise ethnostylistique serait vouée à l'échec, dans la mesure où «la solitude du signe [est] la rançon de l'autarcie du concept» (2006 : 3). Comment, concrètement, relier l'ethnonyme à son contexte culturel, sinon par le cotexte ?

La sémantique interprétative invalide donc l'universalisme sémantique dans lequel le sens est « fixé par un codage préalable [associant] strictement un signifiant et un signifié » (Rastier $2006: 4$ ), si bien que «le contenu du signe n'est pas un concept universel, mais un signifié relatif à une langue " (Rastier [1991] 2001 : 102), ce que confirme l'hypothèse sémantactique selon laquelle un "prisme culturel [filtre] le "cognitif" », impliquant ainsi «la notion d'universaux [...] "localement interprétés" » (Nicolaï 2001 : 33). Ceci nous amène à l'hypothèse de l'argumentativité intrinsèque des mots posée par Raccah.

Sa sémantique des points de vue confirme que derrière les mots, il y a toujours déjà du discours : ils sont « chargés des idéologies qui ont marqué l'évolution de la culture dans laquelle nous vivons et de la langue 
que nous parlons » (Raccah 2002 : 264). Le sémanticien incite donc à prendre en compte « la contribution des mots de langue à l'argumentativité des énoncés [...] par l'attribution de points de vue, "cristallisés" dans ces mots et disponibles par défaut pour construire une orientation argumentative lors de la mise en énoncé ». Ainsi, les mots d'une langue reflèteraient « une part de l'idéologie de la communauté qui parle cette langue» (Raccah 2011a : 124). En effet, outre les mots aux connotations euphoriques ou disphoriques, les mots ordinaires aussi déclenchent des contraintes sur les énoncés : « il s'agit de "miniprogrammes" argumentatifs déclenchés par des mots évoquant des points de vue qui s'appuient sur d'autres points de vue : le jugement évoqué par ces mots [...] est contraint par le jugement que l'interprète porte sur d'autres entités que celle que le mot évoque » (Raccah 2011a : 132). Le rapport entre les mots ainsi mis en contact peut être doxal (l'un confirme l'autre), paradoxal (l'un s'oppose à l'autre), ou adoxal en cas de rapport que le sens intrinsèque des mots ne pouvait laisser prévoir (id., $1990: 196)$.

Si donc la sémantique des points de vue autorise « un traitement sémantique poussé de l'argumentation, à condition d'accorder une certaine attention aux phénomènes lexicaux » (ibid. : 194), alors nous postulons qu'elle ouvre par là-même une entrée lexiculturelle dans un texte littéraire par l'ethnostylistique de l'emprunt. Reste à présent à définir le fonctionnement argumentatif des lexies du corpus : comment l'analyser, et avec quels outils?

\section{Du corpus des emprunts à l'analyse des glossaires}

Comme le remarque Thibault (2012 : 4), un glossaire d'une œuvre francophone « qui réunirait tous les emplois rencontrés dans la littérature et pouvant poser des problèmes de compréhension à un public francophone » exogène, comporterait des termes hétérogènes « du point de vue de l'appartenance des unités traitées à un code linguistique bien déterminé, mais serait en revanche homogène du point de vue du corpus décrit $»$. Les emprunts lexématiques de notre corpus ont été sélectionnés en fonction de deux critères. En langue, leur absence d'un dictionnaire répertoriant les usages du français de référence tel que le Petit Robert va dans le sens d'un statut d'emprunt non intégré. En discours, leur mise en relief autonymique (Authier-Revuz 1995) par des italiques ou par la présence d'une glose métalinguistique est aussi un critère de sélection à prendre en compte.

L'ensemble des lexies sélectionnées par ces deux critères peut donc être figuré par les deux cercles cidessous, la plupart se situant à l'intersection. Estimant que ce recouvrement imparfait des critères d'intégration en langue et en discours présente à l'étude des cas intéressants, nous retenons aussi les termes en marges : ceux qui sont mis à distance par nos écrivains quoiqu'inclus dans le Petit Robert, et à l'inverse, ceux qui, bien qu'absents du Petit Robert, ne sont pas mis en relief par les auteurs. Il faut noter que dans le cas des ethnonymes, le critère lexicographique est plus complexe à appliquer car du point de vue des dictionnaires de lexicographie générale, leur lexicographisation dépend d'une " prise de position théorique sur ces mots qui se trouvent à la limite du nom propre et du nom commun » (Steuckardt 2009 : 91). Quant à l'IFA (Inventaire des particularités lexicales du Français en Afrique noire, [1983] 1988), lorsqu'il les retient, il les répertorie dans une liste à part et non dans le corps de l'inventaire.

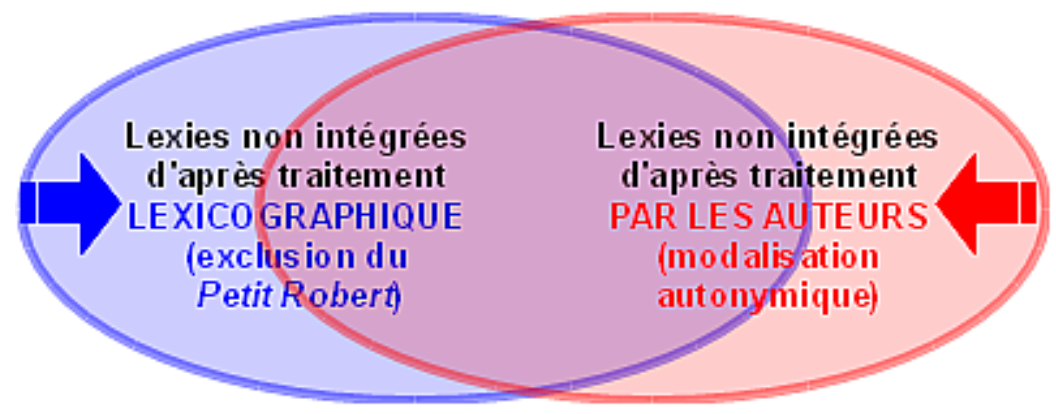

Schéma 1 : sélection des lexies du corpus 
Notre corpus comporte par conséquent 6 items provenant du roman de Sow Fall, déclinés en 115 occurrences, 6 items du roman de Maalouf déclinés en 119 occurrences, et 18 items du roman de Kourouma, déclinés en 111 occurrences. Notre perspective est donc qualitative. Le corpus examiné ici est un fragment d'un corpus plus vaste, de 1303 occurrences relevées dans les trois romans, et correspondant à 175 lexies différentes : 60 chez Sow Fall, 68 chez Kourouma, 47 chez Maalouf. Cet ensemble a été analysé, sous le jour notamment des rapports entre fonctions sociale et expressive-créative, dans un article à paraître chez Fabula-LHT (Schwob 2014b).

À présent, quelle méthode d'analyse adopter ? Nous ne ferons pas œuvre, pour étudier les termes du corpus, de lexicographe, mais nous examinerons leur traitement par les auteurs à la lumière d'outils d'analyse issus de la lexicographie différentielle. Adaptant l'approche interprétative en lexicographie différentielle, Frey (2008a : 30) montre que les emprunts font l'objet d'une évaluation en discours par les locuteurs endogènes ou exogènes, entre «assimilation » et « distanciation». En effet, parmi les critères de sélection des lexies retenus par l'IFA (Bal [1983] 1988 : XIV), la lexicographie interprétative porte une attention particulière au jugement du locuteur sur l'emprunt, aux motivations qui président à son emploi, aux précautions aussi qui permettent au locuteur d'anticiper sur sa réception.

Dans le cadre de cette évaluation des emprunts, on peut donc opposer le cas où l'on voit apparaître les signes d'une prise de distance du locuteur à l'égard du mot emprunté, que ce soit par l'usage en mention du terme et/ou par sa glose, et celui où l'absence de signes, parmi lesquels la glose, est en soi signe d'assimilation du terme. Or, concernant les romans du corpus, nous postulons qu'une analyse similaire peut être menée : en effet, nous estimons que le traitement variable de l'emprunt par la glose suppose, comme reformulation, la prise en compte d'un narrataire doué de compétences linguistiques situables sur un continuum entre le français de référence et ses formes marquées par le contact des langues. De fait, si le « narrataire degré zéro » est supposé comprendre la langue de l'auteur (Prince 1973 : 181), en revanche, les modalisations autonymiques (Authier-Revuz 1995) des emprunts par la glose, impliquent une situation de communication exolingue marquée par le déséquilibre des compétences entre narrateur et narrataire (De Pietro 1988).

Les traitements variables de l'emprunt lexématique par les auteurs pourront donc se décrire par l'opposition entre les modes de communication exolingue et endolingue en situation de contact des langues définie par la linguistique interactionnelle (De Pietro 1988). En effet, la première stratégie, relevant d'une "conception dialogique du discours ", entraîne "des ajustements réciproques des interlocuteurs », alors que dans la seconde, «les divergences codiques » n'étant «plus perçues comme significatives », ne suscitent pas de reformulations (Nicolaï 2000 : 168-169).

En prenant pour point de référence la langue dont vient l'emprunt lexématique, nous pouvons ainsi situer les traitements possibles de l'emprunt dans les romans du corpus entre assimilation et distanciation. Nous qualifierons alors le traitement par assimilation d'endoréférentiel (le traitement du terme implique que le narrataire privilégié est censé comprendre l'emprunt), et celui par distanciation d'exoréférentiel (le narrataire privilégié est supposé ne pas connaître l'emprunt). L'usage des termes sans mise à distance sera à ce titre aussi révélateur que leur emploi en mention autonymique, qui se voit souligné par les italiques et/ou la présence d'une glose. Nous chercherons donc les signes invitant à placer la pratique des auteurs sur un continuum entre assimilation et distanciation.

Outre ces signes, il convient d'analyser de près le fonctionnement de la glose. Sans perdre de vue que le texte littéraire poursuit d'autre finalités que le dictionnaire, nous prendrons pour référence de la prise en charge maximale du lecteur, les articles de lexicographie différentielle. Thibault (2006) inaugure l'étude de la glossairistique des textes littéraires francophones avec un modèle de glossaire philologique pour leur édition, représentant à ses yeux un programme maximal. Dans son article «Glossairistique et littérature francophone ", il analyse les glossaires ajoutés à des textes francophones par les éditeurs, traducteurs ou écrivains, qui, sans être lexicographes de profession, ont le souci de faciliter l'accès de ces œuvres à leurs lecteurs exogènes. Après avoir décrit les lacunes de ces glossaires au regard de la lexicographie différentielle, il présente sa propre vision du glossaire littéraire francophone. 
Sous sa forme maximale, celui-ci comportera notamment les éléments suivants : entrée, catégorie grammaticale, acceptions, fonctionnement syntagmatique, contexte d'apparition, renvoi à d'autres contextes où l'emprunt est présent ; rubrique encyclopédique, remarques formelles, remarques différentielles (lorsque l'analyse de la lexie engage des comparaisons entre le français diatopiquement marqué et le français de référence) ; commentaire historico-comparatif retraçant l'histoire du terme et le mettant en perspective dans le cadre plus large de la francophonie ; bilan bibliographique.

Nous cherchons donc à éclairer la pratique glossairistique singulière des auteurs à la lumière de ce modèle, perçu comme un idéal régulateur. Ce faisant, nous prenons au sérieux la constitution de glossaires et les propos quelquefois explicites des écrivains se situant par rapport à l'entreprise dictionnairique. La glose maximale présenterait les éléments répertoriés dans le tableau ci-dessous.

\begin{tabular}{|l|c|c|c|}
\hline & Sow Fall & Kourouma & Maalouf \\
\hline Entrée & + & + & + \\
\hline Catégorie grammaticale & $+/-[+10]$ & - & - \\
\hline Acception & $+/-[-2]$ & + & + \\
\hline Fonctionnement syntagmatique & - & - & - \\
\hline Autres contextes & - & - & $+/-[+2]$ \\
\hline Rubrique encyclopédique & - & $+/-[+8]$ & $+/-[+2]$ \\
\hline Remarques formelles & - & - & $+/-[+4]$ \\
\hline Remarques différentielles & - & $+/-[+4]$ & $+/-[+1]$ \\
\hline Commentaire historico-comparatif & - & $+/-[+1]$ & $+/-[+3]$ \\
\hline Bilan bibliographique & - & $+/-[+20]$ & $+/-[+2]$ \\
\hline Total des rubriques représentées & $\mathbf{3}$ & $\mathbf{6}$ & $\mathbf{8}$ \\
\hline Écart / article lexicographique & $\mathbf{7}$ & $\mathbf{4}$ & $\mathbf{2}$ \\
\hline
\end{tabular}

Tableau 1 : représentation des rubriques d'un article de lexicographie différentielle dans les gloses

Nous notons la présence systématique d'une rubrique par +, l'absence systématique par -, sa présence occasionnelle par +/-, avec entre crochets le nombre d'apparitions de la rubrique. Nous enregistrons la présence de certaines rubriques même si elles n'existent qu'à l'état embryonnaire par rapport au modèle de Thibault. Si les rubriques systématiquement ou occasionnellement présentes dénotent en creux la constitution d'un glossaire dans le roman, nous pouvons alors confronter les pratiques glossairistiques des trois auteurs au programme maximal de l'article lexicographique en termes de diversité des rubriques représentées $^{2}$. Nous nous reporterons à ce tableau pour chaque cas, en élargissant alors nos analyses aux 1303 lexies du grand corpus.

\section{Sow Fall et le "Dioula que personne ne connaît »... Des ethnies à nommer}

Dans Le Jujubier du patriarche de Sow Fall, les ethnonymes sont assez rares : on trouve Dioula en deux occurrences (Sow Fall [1993] 1998 : 24, 70), Mandingue en une seule (ibid: 166). Aucune de ces lexies n'est mise à distance dans le texte, ni par les italiques ni par la glose. On rencontre également l'ethnonyme informel toubab en trois occurrences (ibid : : 64, 84, 117). Celui-ci est mis en italiques mais n'est pas glosé. Ce terme est assez polysémique ; l'IFA ([1983] 1988 : 372) l'explicite, en première acception, comme « toute personne ayant la peau blanche, à l'exclusion des Arabo-Berbères », et c'est en ce sens que ce terme est employé dans le roman. Si Diop (2003 : 119) nous apprend que le lieu symbolique du retour 
aux sources dans l'intrigue, Babyselli, correspond à Seylibabi, en territoire mauritanien, situé «au carrefour de plusieurs influences culturelles et linguistiques : toucouleur, maure, saraxolé, peul, wolof », il est par ailleurs frappant de constater que la seule allusion explicite à l'une de ces ethnies se fait à travers le glottonyme wolof, utilisé pour gloser un anthroponyme individuel, Goudi (Sow Fall [1993] 1998 : 57). Nombreuses sont en revanche les périphrases destinées à nommer les clans en présence, telles le peuple du turban, ou le clan des chasseurs-magiciens. Pour les comprendre, il importe de connaitre l'épopée du Foudjallon, intertexte omniprésent du récit, qui célèbre implicitement la synthèse de ces clans rivaux, musulmans et païens (Guèye 2005 : 157-158).

Quant aux noms de représentants des pouvoirs temporel et religieux, ils sont assez rares aussi. Nous trouvons linguère, en deux occurrences (Sow Fall [1993] 1998 : 37), sans glose mais en italiques, qui dénote la « dame de la cour royale du Kajoor », ancienne région du Sénégal (Diop : 339); et Damel (Sow Fall [1993] 1998 : 22), en une occurrence, ni glosée ni marquée. Ce terme correspond au «titre porté par le souverain du Kajoor » (Diop : 140). Il porte une majuscule car il correspond dans le roman à la lignée du même nom, les « descendants des Damel » : anthroponymes collectifs et individuels se rejoignent ici. Enfin, Almamy, ni marqué ni glosé, se trouve en 106 occurrences dans le texte. Il s'agit d'un «chef de prière » (Diop : 35). Il porte toujours la majuscule et se rencontre au singulier, ainsi qu'au pluriel sans $-s$ (les Almamy). Il partage avec le prénom Yellimané, un des personnages principaux du récit, descendant de l'Almamy, une étymologie commune, al imam, qui indique son lien avec la religion musulmane : cette observation confirme la prégnance, dans le corpus, des liens entre anthroponymes collectifs et individuels, liens qui ne peuvent être mis en évidence qu'à partir d'une connaissance du fonctionnement des langues d'adstrat, elles-mêmes constituées à partir d'autres langues prêteuses, comme ici, l'arabe.

De ces observations, il découle que l'emploi des ethnonymes et représentants du pouvoir fait l'objet d'un certain implicite pour le lecteur exogène. Cet implicite reflète la connivence que ce récit des origines cherche à établir entre les Sénégalais, connivence horizontale, au présent, et plongeant ses racines dans la verticalité du passé, grâce à la réinscription de la généalogie épique dans le roman : Le Jujubier du patriarche est, comme l'explique Guèye, le récit d'une synthèse. C'est autour de la redécouverte de ce rapport de descendance qui transcende les conflits interethniques du présent, que se déploie la narration. Dans ce contexte, personne, dans l'expression « un Dioula que personne ne connaît ( (Sow Fall [1993] 1998 : 70), se comprend comme «personne d'entre nous » : cet ethnonyme n'a pas besoin d'être glosé pour les lecteurs endogènes, justement unis par la connivence et la connaissance réciproque. On le voit, cette connaissance se manifeste notamment à travers les noms de clan, comme Damel, qui se confondent avec les ethnonymes individuels dans un rapport métonymique renforçant par l'onomastique le sentiment de partage.

Le traitement de tous ces termes, on le constate, oscille entre assimilation et distanciation ; le tableau décrivant la pratique glossairistique des auteurs révèle que la représentation des rubriques du glossaire est minimale : la plupart des termes de notre corpus ne sont pas glosés, et quand ils le sont, c'est seulement pour en révéler l'acception. En revanche, la référence à la catégorie grammaticale reflète l'importance prise par les interjections qui semblent présenter un vide référentiel si elles ne sont pas interprétées en contexte. Là aussi, la condition de l'interprétation des emprunts est dans la connivence d'une situation partagée ${ }^{3}$. Il faut noter que la longueur moyenne des gloses dans le roman est de 1717 caractères, ce qui est assez peu en comparaison des deux autres œuvres du corpus.

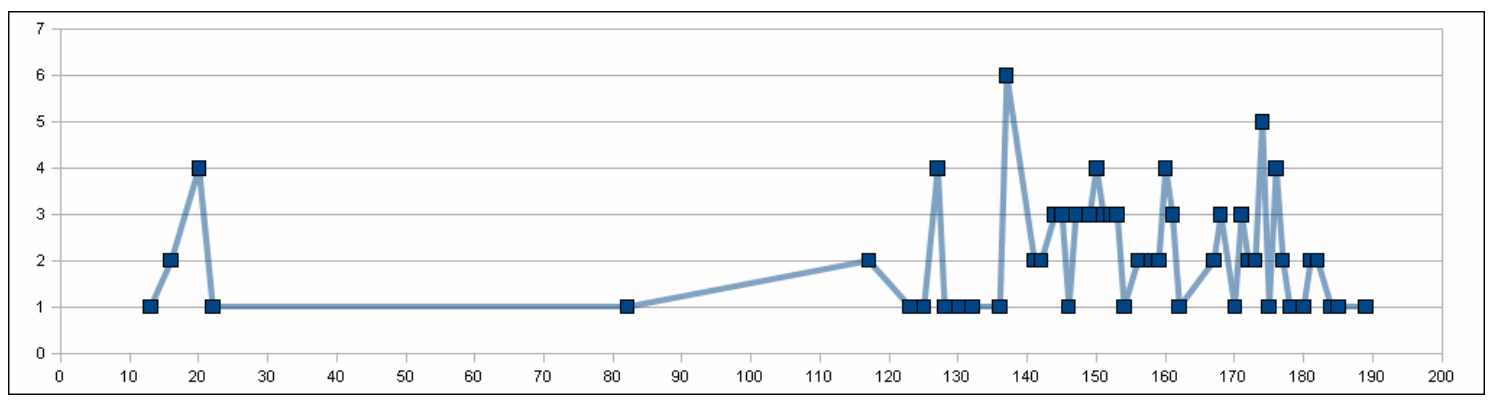




\section{Graphique 1 : occurrences de Almamy chez Sow Fall}

Le graphique ci-dessus présente la répartition des occurrences d'Almamy dans le récit. On remarque leur forte concentration au moment où le roman, vers son centre, bascule vers l'épopée et la découverte du passé fondateur, avec la mise en valeur de l'ancêtre commun.

\section{$5 \quad$ Avec Kourouma, à chacun ses Bambaras... « aussi appelés Lobis, Sénoufos, Kabiès, etc. »}

C'est dans Allah n'est pas obligé que les ethnonymes sont les plus nombreux : Bambara, Dioula, Guéré, Gyo, Kabiès, Krahn, Lobi, Malinké, Mandingo, Mendé, Ogoni, Sénoufo, Temné, Timba, pour un ensemble de 87 occurrences. Ces termes représentent une partie de la diversité des ethnies que Birahima, le narrateur, peut croiser dans son périple entre Libéria et Sierra Léone. Aucun des emprunts du roman n'est marqué typographiquement ; le niveau métalinguistique des gloses est en général marqué par des parenthèses. En revanche, ce qui caractérise le traitement de ces termes, c'est la récurrence fréquente des gloses qui leur sont consacrées. On constate dans le graphique ci-dessous que Malinké, en 40 occurrences, est glosé 11 fois, quelquefois à deux reprises dans la même page.

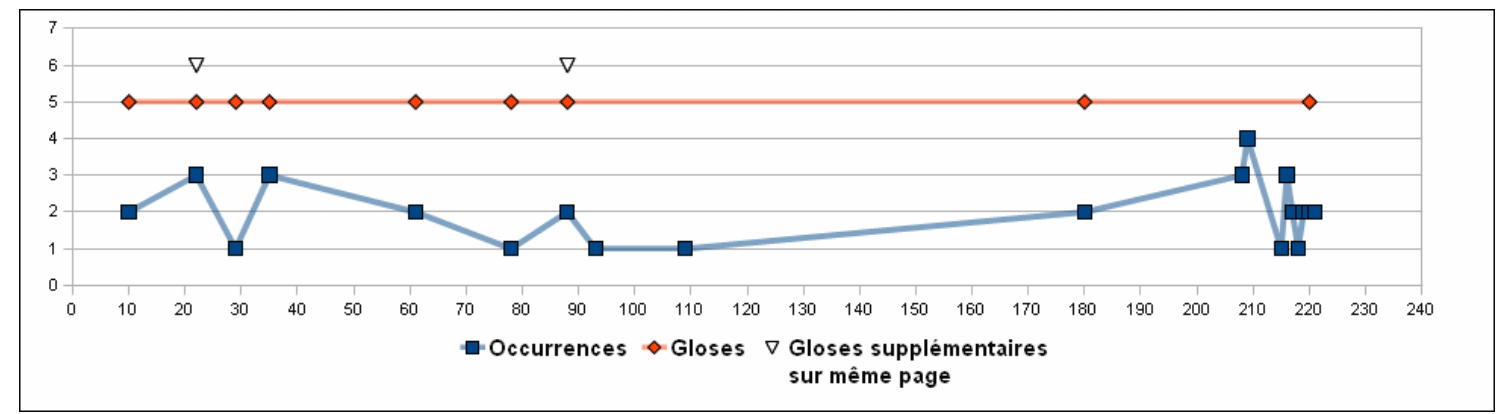

Graphique 2 : occurrences et gloses de Malinké chez Kourouma

Beaucoup de ces gloses ont une dimension axiologique, ainsi « les Malinkés sont des étrangers ; ils sont venus de la vallée du Niger depuis longtemps et longtemps. Les Malinkés sont des gens bien » (Kourouma 2000 : 22), ou encore : "les Malinkés sous leurs grands boubous paraissent gentils et accueillants alors que ce sont des salopards de racistes » (ibid. : 61). Les gloses de Kourouma jouent donc sur le stéréotype ethnique, en tant que « représentation concernant un groupe et ses membres » (Amossy 1997 : 35), dont l'évocation «se déploie selon une opposition binaire du type bon/mauvais, musulmans/[...] sauvages »; mais le narrateur montre que si, « dans la mentalité mandingue, les autres sont des féticheurs, des méchants », toutefois, "malgré cette vision du monde, les malinké, dans un syncrétisme religieux total pratiquent la religion musulmane et ont cependant recours aux croyances magico-religieuses » (Barry $2006: 10)$. Cette stratégie de redéfinition permet un renversement des points de vue, montrant la réversibilité des connotations que ce terme peut prendre. Pour un lecteur favorable à l'ethnie malinké, la glose peut sembler paradoxale, au sens que Raccah donne à ce terme.

Kourouma use aussi d'ethnonymes de façon plus informelle, avec toubab et Bushmen. Selon la même logique que Malinké, toubab est glosé six fois pour neuf occurrences. Bushmen prend ici le sens informel d'une insulte, c'est donc plus pour ses connotations qu'il est choisi. L'intertexte avec le dictionnaire étant une caractéristique prégnante du roman, Kourouma (2000:35) cite d'abord une définition de l'IFA, à sa manière : «Bushmen signifie, d'après Inventaire, hommes de la forêt, nom donné par mépris par les hommes de la savane aux hommes de la forêt ». Birahima glose une nouvelle fois le terme (ibid.), dans ses propres mots : "Les Bushmen sont des gens de la forêt qui ne sont pas malinkés et qui ne connaissent pas la circoncision et l'initiation ». La stratégie de Kourouma consiste à dévoiler le point de vue qui est à l'origine des traits sémantiques attribués aux «Bushmen » dans le cadre d'une définition négative, l'ironie soutenant l'intention satirique du roman. 
Quand aux Bambaras, la glose fait décidément apparaître le caractère plus que flottant de ce signifiant, en même temps que les apories auxquelles aboutissent les tentatives de catégorisation coloniale, qui révèlent aussi la méconnaissance dont ils font l'objet : «les Bambaras sont parfois aussi appelés Lobis, Sénoufos, Kabiès, etc. Ils étaient nus avant la Colonisation » (ibid. : 22). Au-delà de l'ironie savoureuse en fin de glose, l'auteur souligne, comme le fait Froidevaux à la suite de Bazin dans un article dont nous empruntons le titre pour cette section ${ }^{4}$, que «c'est à la suite d'une série de confusions et de définitions approximatives que l'ethnie bambara est apparue dans la littérature ethnographique » pour être « recensée en tant que telle par l'administration coloniale » (Froidevaux 2005 : 71). Pour suggérer l'impossibilité de définir, l'accumulation des ethnonymes supposés en coréférence est efficace, stratégie familière à Kourouma, comme le note Caitucoli : « un autre moyen pour faire perdre au mot ses connotations, c'est l'accumulation de synonymes. En usant plusieurs synonymes pour exprimer une réalité ou un sentiment, l'écrivain ex-colonisé veut souvent signaler l'approximation du sens des synonymes à saisir la réalité » (2007 : 60). L'étoffement par la glose suggère ainsi l'absence de bijection entre deux langues. Par ce geste notamment, Kourouma dévoile que ces ethnonymes, à l'uniréférentialité desquels le lecteur pourrait croire, ont une signification flottante. Au-delà de leur fonction cognitive, il met ainsi en valeur leur fonction sociale, l'usage d'un terme ou d'un autre révélant un point de vue sur les peuples, voire une appartenance du locuteur qui les emploie.

Parmi les désignations du pouvoir temporel ou religieux, nous retrouvons almamy, en une occurrence, glosé et sans majuscule (ibid. : 75), ainsi qu'imam, glosé une fois pour neuf occurrences. Kourouma est conscient de l'intégration de ce terme dans le lexique du français de référence, il ne le glose d'ailleurs qu'en sa deuxième occurrence, sept pages après la première, geste exceptionnel chez lui : " c'est ainsi on appelle $[s i c]$ le vieillard à la barbe blanche qui prie devant tout le monde le vendredi, les jours de fête et même cinq fois par jour » (ibid. : 30). Cette définition voltairienne, en associant la prière à un spectacle, vide évidemment le terme de toute connotation spirituelle. Le terme a donc, certes, une fonction cognitive dominante ; mais par sa redéfinition il acquiert aussi une dimension clivante en suggérant le dissensus sur les croyances. Les doubles définitions mettent ainsi en évidence la présence de points de vue et de discours polyphoniques derrière les termes employés.

On remarque, d'après le tableau des pratiques glossairistiques des auteurs, que les rubriques encyclopédique, différentielle et bibliographique (modestement présente par la référence aux ouvrages lexicographiques) sont assez souvent représentées. La longueur des gloses (12625 caractères sur tout le roman), la comparaison du sens d'un terme supposé équivalent en français et dans les langues d'adstrat, l'ampleur des passages encyclopédiques, attestent d'une volonté d'adopter un point de vue émique sur les cultures décrites. La référence au dictionnaire permet d'opposer une glose à la subjectivité assumée à une glose censément objective. Sur ces points, le traitement des noms de représentants du pouvoir est en cohérence avec celui des ethnonymes.

\section{Maalouf et «ces diables d'Ankliz » : pour une anthropologie littéraire émique}

Dans Le Rocher de Tanios, nous ne trouvons qu'un ethnonyme qui soit un emprunt non intégré : Ankliz, en deux occurrences ([1993] 2004 : 101, 113). Ce terme désigne évidemment les Anglais. Si cet ethnonyme lexématique est quasiment un hapax, il a le mérite d'être spectaculaire, dans la mesure où le français de référence dispose de toute évidence d'un terme dénotant cette nationalité : pour le coup, ce référent n'est pas original à la culture libanaise. Quant aux désignations de représentants du pouvoir religieux ou temporel, nous trouvons cheikha, en 32 occurrences, pendant féminin de cheikh, recensé quant à lui en lexicographie générale ; khourriyyé, en 19 occurrences, glosé par «l'épouse du curé » (ibid. : 46). Nous rencontrons aussi des cas plus particuliers, avec les appellatifs bouna, équivalent de "père », en 32 occurrences, et sayyedna, en 8 occurrences, employé dans un sens proche de bouna, mais en coréférence au Patriarche ; enfin, le cas de beyk, en 26 occurrences, intégré dans le Petit Robert mais exotisé par une graphie conservatrice. Ces lexies reçoivent des traitements divers. Beyk n'est ni marqué ni glosé. Cheikha n'est pas marqué, mais glosé. Ankliz est marqué par des guillemets sans glose. Tous les autres termes sont mis en italiques et glosés. 
Par rapport aux autres auteurs, l'usage des ethnonymes et des toponymes par Maalouf est immédiatement argumentatif, en l'absence de toute glose, de façon spectaculaire. En effet, Ankliz implique un renversement des points de vue de l'Occident vers l'Orient, comparable à celui suscité par l'ethnonyme Franj dès l'avant-propos des Croisades vues par les Arabes (1983 : 5) : « le mot qui désigne les Francs est transcrit différemment selon les régions, les auteurs et les périodes : Faranj, Faranjat, Ifranj, Ifranjat... Pour unifier, nous avons choisi la forme la plus concise, celle surtout qui sert aujourd'hui encore dans le parler populaire à nommer les Occidentaux, et plus particulièrement les Français : Franj ». On retrouve le même procédé avec l'emploi du toponyme Roumieh, "la ville du pape » ([1993] 2004 : 57). Quoique le même référent soit visé, la conception du référent change avec le nouveau signifiant, et c'est si peu anodin que l'emploi d'un terme ou d'un autre finit par définir, ou confirmer, des groupes ou l'appartenance des individus à des groupes. Il est patent que l'emploi même de ces mots implique des contraintes sur le discours littéraire qui les environne : ils sont, d'emblée, perceptibles comme de mini-programmes argumentatifs.

En ce qui concerne les désignations de représentants du pouvoir religieux ou temporel, Maalouf semble employer différents procédés visant à l'exotisation des termes, afin de pousser le lecteur à une interprétation non réductrice des lexies. Ainsi, cheikh est accompagné de cheikha qui remplace la périphrase «épouse du cheikh » et fait sentir, comme dans le cas de khourriyyé, le pouvoir de l'épouse du dignitaire. Surtout, l'attribut du cheikh, sa main (kaff), qui a le double privilège de la sanction et de la caresse dans le cadre d'un pouvoir patriarcal fortement idiosyncrasique, est très longuement développé à travers la glose narrativisée de kaff (ibid. : 18-20). Dans la suite du roman, le doublet main, lorsqu'il apparaît, est resémantisé avec les valeurs associées de kaff. La graphie conservatrice de beyk peut aussi être interprétée comme procédé d'exotisation, en quelque sorte gage d'authenticité. Bouna, au retour très fréquent comme en témoigne le graphique ci-dessous, est assez curieusement choisi de préférence à khourry, masculin de khourriyyé, disponible en arabe dialectal libanais, خُوري (Sultani \& Milleli : 219), et par lequel le lecteur exogène remonterait de façon assez transparente à l'étymon curé. Comme Roumieh, bouna vise sans doute à faire sentir la différence entre le statut de prêtre catholique et celui de prêtre orthodoxe, et avec elle, la distance entre deux cultures.

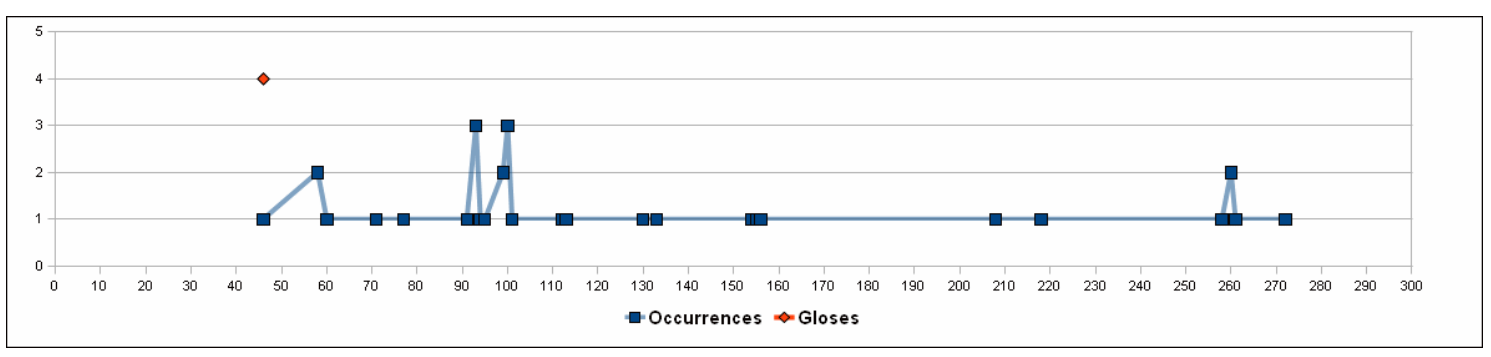

Graphique 3 : occurrences et gloses de bouna chez Maalouf

Ainsi, Maalouf et Kourouma ont chacun leur technique pour désambiguïser les ethnonymes. Ils rendent par là sensible le fait que certains termes «considérés intraduisibles et qui, par conséquent, peuvent être transférés non traduits dans la langue d'arrivée - emprunts et xénismes - constituent en réalité des traductions en raison du glissement sémantique qui accompagne systématiquement leur réception et leur intégration dans la culture d'accueil » (Durieux 2010 : 23), avec notamment une réduction des champs lexico-sémantiques et des nuances de sens. Ceci est confirmé par Queffélec, pour qui « le bilinguisme des locuteurs confère [aux mots-voyageurs] une richesse dénotative que ne leur connaît pas le français central qui les a empruntés à date plus ancienne [...] et a réduit leur polysémie à une simple monosémie » (Queffélec 1998 : 253-254). L'exotisation permet ainsi de corriger la portée connotative du mot, l'évitement de la traduction-réduction par les locuteurs bilingues étant le signe de la plus-value connotative de ces termes.

Comme chez Kourouma, l'emploi de ces termes indique donc chez Maalouf une appartenance et remplit 
ainsi une fonction sociale. Mais, même dans le cas de doublets patents comme Ankliz et Roumieh, les termes semblent remplir aussi une fonction cognitive, qui amène à repenser les connotations du signifié, et à changer de point de vue sur les cultures et leurs rapports. Si l'usage émique du mot juste, avec un changement de point de vue qui peut être souligné par la glose, implique le parti-pris qui renvoie l'usage de ce terme et son traitement à la fonction sociale, ce changement de point de vue, en retour, active également la fonction cognitive en incitant le lecteur exogène à changer de regard sur le référent préalablement identifié, par une réinterprétation du signe en fonction d'un cotexte, et d'un contexte qu'on ne partageait pas mais qu'on apprend à partager, en vertu de la dimension didactique de la littérature interculturelle. Maalouf est celui qui exploite le plus les potentialités du glossaire littéraire : par ailleurs, sur tout le roman, ses gloses représentent 10395 caractères. La longueur et la précision de certaines leur prête une dimension encyclopédique. Les remarques différentielles et l'allusion à d'autres contextes où sont employés les termes (c'est le cas notamment pour kaff) ne peuvent que développer chez le lecteur une sensibilité accrue aux connotations des termes.

Il est donc clair que « le choix d'un ethnonyme plutôt qu'un autre pour un même groupe se justifie par la volonté de préserver le point de vue de l'interlocuteur ou du groupe dont on parle ». En effet, l'utilisation des ethnonymes lexématiques procède de "la nécessité de faire une distinction entre deux termes apparemment équivalents, mais qui recèlent en réalité deux significations différentes, voire qui indiquent deux groupes dont les frontières et les critères de classement [...] peuvent être partiellement divergents » (Ciavolella $2008:$ 11).

Si Ankliz ou Roumieh sont des mini-programmes argumentatifs, c'est aussi en ce que, selon l'expression de Benzakour (2000 : 322), en tant que mots-scénarios, ils cristallisent une Histoire et stockent des points de vue, des discours sur cette Histoire. Ils ont alors, comme les désignants d'événements, « la capacité de stocker les coordonnées de l'événement, et en conséquence d'éveiller la mémoire des faits par la seule mention du "nom" ». Dans les cas où cette Histoire n'est pas connue du lecteur exogène, l'auteur a la possibilité de gloser les termes : de fait, «lorsque ce type de "nom" est étudié dans un contexte plus large que celui d'une seule langue, son statut devient plus complexe, il n'est plus le même. Pour passer d'une langue à l'autre, l'énonciateur fera un choix qui ne sera pas sans rapport avec sa prise de position quant à l'événement » (Dahech $2013: 7)$.

C'est peut-être alors un des privilèges de la littérature que de rendre partageable, communicable, l'expérience humaine fortement idiosyncrasique. Le roman de Maalouf particulièrement peut être considéré comme la traduction de ce principe prégnant de l'onomastique : «des métonymies se croisent en tous sens dans cet espace élu par un mot. L'homme y devient lieu et le lieu y devient événement» (Cheriguen 1994 : 94), d'où la pertinence d'une stylistique des lieux-sources. En effet, Le Rocher de Tanios est une glose géante du toponyme éponyme : le roman raconte comment on a nommé Tanios, comment, à partir de ce nom et du secret qu'il révéla sur sa naissance, Tanios a vécu, comment cette histoire a modifié l'Histoire, comment le rocher fut nommé et devint dans l'espace de la diégèse le monument de cette Histoire. Les métonymies décrivent le rapport associatif, difficilement assignable pour le locuteur exogène, qui lie une entité à son environnement, faisant voir combien «ces "choses" prétendues sont le résultat d'une objectivation dont on oublie qu'elle résulte d'un couplage entre l'individu et son environnement» (Rastier $2006: 7$ ). L'analyse des ethnonymes et de leur traitement confirme combien ils sont révélateurs de l'environnement politique au sens large, en vertu du lien métonymique qui unit ces termes par des rapports de contiguité dans l'espace-temps géographique et historique d'une communauté, les noms propres disant ce qui est propre à une communauté. Qui de mieux placé que l'écrivain pour reproduire ce bain linguistique, fait d'une vie passée ailleurs, à s'imprégner des opinions et expériences locales, par le détour d'un récit ?

\section{$7 \quad$ Pour conclure : les interactions entre lexicographie et littérature}

En définitive, les pratiques glossairistiques des écrivains ressemblent fort, quelquefois, à des prises de position, non seulement sur le lexique, mais, et c'est patent chez Kourouma, sur l'entreprise dictionnairique elle-même. Benzakour (2010:7) rappelle que bien souvent, «les emprunts ne trouvent place dans les nomenclatures des dictionnaires de référence » qu'à la condition de ne pas être des 
doublets. Quant aux définitions, elles peuvent quelquefois se réduire à la dimension dénotative des lexies recensées, ce que notent Raccah et al. : « ce qui est systématique, dans la description des dictionnaires, c'est l'aspect dénotationnel, (objectif) tandis que les aspects subjectifs des mots de la langue, eux, ne sont pas traités de manière systématique. Les linguistes/lexicographes décrivent les aspects subjectifs apparaissant dans les énoncés, mais ils ne font pas [toujours] ${ }^{5}$ l'abstraction et l'abduction nécessaires pour décrire ces aspects en langue » (Raccah, Ben Mahfoudh-Hubert, \& Chmelick 2011 : 122).

Ainsi, le déploiement des connotations rend le texte littéraire plus proche d'un inventaire que d'un dictionnaire, si on suit les propos de Benzakour (2010:8), qui oppose ainsi ses «définitions "lexiculturelles" » à celle des ouvrages de lexicographie générale : "la définition linguistique ne doit s'intéresser qu'à ce qui est utile au fonctionnement de la langue décrite; elle ne doit donc pas se soucier de ce qui est nécessaire à la connaissance exhaustive du référent du mot à définir », si bien que "si la définition dépasse la limite des traits spécificateurs, en donnant par exemple une description trop détaillée du référent, elle devient encyclopédique » (ibid. : 5-6)..., mais par là, propre à traduire adéquatement la charge connotative des mots. Comme elle, Frey n'a pas craint de «brouiller (mais ne l'est-elle pas de toutes façons, à quelque degré ?) la frontière entre dictionnaire de langue et dictionnaire encyclopédique », de façon à ce que l'inventaire soit, aussi fidèlement qu'il se peut, « le reflet linguistique d'une situation socioculturelle ». Accepter cette modification de l'article lexicographique - loin d'être anodine -, «c'était franchir une étape de l'histoire de la linguistique, en passant du signe saussurien aux approches référentielles, en allant donc de la structure vers la culture » (2008: 245-246). Au fond, le trajet accompli par ces lexicographes est ici défini en termes assez proches de celui de Mendo Zé et rejoint aussi le cheminement des auteurs du corpus. Ces auteurs et chercheurs affirment ainsi, chacun à leur manière, la vocation épistémologique d'une «sémiotique des cultures », démontrant que « comprendre la médiation sémiotique reste indispensable pour décrire les facteurs culturels dans la cognition, jusqu'ici gravement sous-estimés par les recherches cognitives » (Rastier 2006 : 11), ce qui donne à réfléchir sur les liens complexes entre fonctions sociale et cognitive dans l'emploi de l'emprunt ${ }^{6}$.

Cette étape n'est pas sans conséquences sur la conception de l'aventure lexicographique en général. Comme le suggère Benzakour, « toute définition lexicographique est censée viser l'objectivité. Mais elle peut fort bien présenter une subjectivité relative » (2010:3), étant donné qu'elle «n'est pas indépendante de l'environnement socio-culturel et idéologique » (Frey $2008: 243$ ). Or, la distinction entre idéologie et connaissance ne se fait pas sur un plan linguistique mais en fonction de la situation, car il ne peut pas y avoir « de distinction linguistique entre idéologie et connaissance : il n'y a pas de marqueur linguistique d'idéologie (ni de connaissance)» (Raccah 2011a : 137). Le rapport à la référence décrit dans ces analyses sémiotiques amène à renouveler le regard sur le lexique comme institution, dans les dictionnaires et dans les textes littéraires. La glossairistique mise en place par les écrivains peut être considérée comme un commentaire de la lexicographie générale et différentielle, commentaire pris en charge, avec d'autres méthodes, par certains courants de la lexicographie elle-même. Comme le souligne Calvet, évoquant le pacte linguistique original engagé dans l'incipit d'Allah n'est pas obligé, « la littérature est parfois plus clairvoyante que les sciences humaines, et ces quelques lignes [d'incipit] évoquent dix fois mieux que n'importe quel texte de linguistique une niche écolinguistique particulière » (2006:57). Cette remarque décrit des rapports entre linguistique et littérature assez comparables à ceux évoqués par Bergounioux lorsqu'il montre que la parole intérieure pourrait bien avoir été représentée en littérature «bien avant d'être désignée dans les sciences humaines » $(2013: 5)$.

La tentation légitime du développement encyclopédique des dictionnaires laisse envisager la place particulière et complémentaire du roman francophone dans le traitement des mots culturels, stratégie de compensation à la non-nativité. La littérature romanesque francophone est peut-être l'art de raconter, de façon didactique ou connivente, comment on en est venu à voir sous les mots des choses, voire d'autres choses et met en évidence l'entreprise générale de toute littérature. L'auteur peut, comme Maalouf, pratiquer un hétérolinguisme étiologique, et, en racontant l'histoire des noms, remonter à l'événement fondateur et idiosyncrasique dans une poétique du dévoilement : "on pourrait ainsi, en suivant la substitution des dénominations et des langues, raconter l'histoire du monde » (Cheriguen $1994: 98$ )... Le lecteur pourrait alors, de même que le lexicographe tenté par l'encyclopédie, «sentir le pays derrière les 
mots » (Frey 2008b : 245), et peut-être le critique y trouverait-il le centre de gravité de certains récits. Ainsi, d'une manière générale, la réflexion sur le langage des auteurs hétérolingues est dévoilée par la glossairistique engagée chez chacun. L'ethnostylistique oblige à une attention renouvelée aux mécanismes non évidents de la référence à travers la traduction de termes faussement universaux ou délibérément opacifiés : le terme emprunté doit être rapporté à un contexte culturel d'énonciation pour être compris, comme dans une littérature illusoirement monolingue d'ailleurs ${ }^{7}$.

Nous pensons que ces réflexions offrent la perspective d'une mise en place d'une approche structurale qui permette de situer les auteurs dans un système d'oppositions maniable et transférable à d'autres auteurs, en vue d'une poétique interculturelle comparée à partir de la glossairistique, dont le tableau reproduit supra, qui met en évidence les rubriques exploitées dans le cadre d'un traitement entre assimilation et distanciation, est une illustration possible. En effet, la suite syntagmatique formée par l'emprunt et sa glose entrent dans le cadre d'un système oppositif mettant en jeu le dialogue entre norme et variation. L'ethnostylème entre dans un rapport oppositif paradigmatique : soit il pose une absence dans la langue cible, liée à l'originalité du référent, et à ce titre il fait signe vers une autre culture ; soit comme doublet, il comporte d'autres virtualités combinatoires, s'intègre dans d'autres champs lexico-sémantiques, et à ce titre, fait là encore signe vers le système de la langue-culture en entier. Dans les deux cas, il convoque donc le système oppositif de la langue et les contacts entre les langues-cultures, et s'intègre par là dans le cadre d'une analyse structuraliste, gage de scientificité.

Notre analyse visait à montrer comment, dans des énoncés doxaux, adoxaux ou paradoxaux, les écrivains se situent par rapport au programme de contraintes de l'emprunt sur leurs phrases : en quelque sorte, l'analyse de discours finit par s'intégrer dans la lexicographie, et ce mouvement est explicité par la sémantique des points de vue, qui décrit les mots comme des concentrés de discours renvoyant à une unité plus antérieure et moins minimale, le discours social. La façon de gloser ces emprunts indique également la constitution d'un pacte linguistique entre norme et variation, évaluable en termes de signes oppositifs. L'analyse du sens global de l'œuvre et des propos des écrivains concernés est une procédure de confirmation des hypothèses réalisées sur ce pacte linguistique. L'intertexte littéraire, plus ou moins conscient et développé, avec les pratiques glossairistiques et dictionnairique amène à renouveler le regard sur le lexique en tant qu'institution par les œuvres littéraires et les dictionnaires, et offre la perspective d'une entrée nouvelle, de nouvelles catégories et méthodes d'analyse de la littérature en général.

En effet, comme la citation de Calvet (supra) le laisse comprendre, le traitement de l'emprunt lexématique par la glose dans le cadre de romans hétérolingues permet d'entrevoir de nouvelles possibilités d'analyse concernant le pacte littéraire (Baroni 2004), envisagé ici comme pacte linguistique engagé entre narrateur et narrataire. La contribution de la linguistique interactionnelle à l'étude de ce pacte littéraire semble à cet égard prometteuse en ce qu'elle permet d'analyser le traitement des emprunts lexématiques sur un axe entre deux pôles opposés, assimilation et distanciation. En effet, d'une part, l'absence de reformulation placerait le narrataire exogène en position d'acquisition incidente du lexique des emprunts, car leur apprentissage, s'il a lieu, s'effectuerait comme «"produit-dérivé" d'une autre tâche », la lecture (Hulstijn 1994 : 79). D'autre part, par contraste, l'appareil des gloses s'envisagerait quant à lui comme la marque d'un «contrat didactique » (De Pietro, Matthey \& Py 1988) propre à orienter l'acquisition du lexique endogène. Ce contrat didactique, comme nous avons pu le voir, ne concerne pas seulement l'acquisition d'un lexique, mais d'une lexiculture (Galisson 1988), le traitement des ethnonymes permettant tout particulièrement à l'auteur de mettre l'accent sur le lien indissociable entre langue et culture ${ }^{8}$.

Cette approche nous semble propre à renouveler l'analyse du pacte linguistique engagé en littérature : il s'avère possible de le décrire ici par référence au contrat didactique, dont l'absence même révèle un positionnement de la part de l'auteur'. Nous estimons en effet que l'absence de contrat didactique peut s'interpréter de diverses manières, et se comprendre comme une inscription particulière de l'auteur dans les champs littéraires francophones, en résonance avec les problématiques postcoloniales. Ainsi, l'écriture connivente de Sow Fall, caractérisée notamment par une pratique assez parcimonieuse de la glose, pourrait-elle suggérer un positionnement dans le cadre nouveau du para-colonial tel que le définit Newell. Celle-ci, comme le montre Diouf (2006 : 32), « affirme avec force que la meilleure manière d'analyser les 
négociations très complexes qui sont menées au niveau local, relativement à la transmission culturelle est d'adopter un point de vue non colonial», si bien que «le préfixe "para" souligne toute l'ambiguité des réseaux et formules culturelles de l'Afrique occidentale coloniale en signifiant un en-deçà (beside) et un au-delà (beyond) de la domination coloniale et des transactions culturelles qui en dérivent ». Les choix linguistiques de Sow Fall sont donc à corréler à des enjeux littéraire et politique forts, les deux étant indissociables dans le cadre d'analyse des études postcoloniales. Dans le cas de Kourouma et Maalouf, des analyses comparables peuvent être menées. Ainsi, concernant Kourouma, Calvet (2006 : 61) interprète le pacte linguistique particulier engagé dans Allah n'est pas obligé comme le symbole d'une position intermédiaire entre normativisme et variationnisme, manifestée, entre autre, par le système des doubles gloses, et par la relecture des mots du point de vue exogène du français de référence mais aussi du point de vue des locuteurs endogènes : si « entre ces deux termes oppositifs il devrait être possible de trouver un moyen terme », c'est précisément la détonante pratique kouroumienne de ce «moyen terme » qui, à ses yeux, représente l'espoir d'un « ferment de liberté linguistique, un ferment de diversité, c'est-àdire au bout du compte un ferment de démocratie ». Cette position nouvelle peut également se comprendre comme une autre forme de recherche de dépassement des oppositions traditionnelles imposées par l'histoire ${ }^{10}$. Nous avons aussi pu montrer (Schwob 2014b) comment la pratique didactique de la glose par Maalouf, qui peut s'interpréter comme posant les cadres d'une acquisition fortement guidée pour le lecteur exogène, manifestait un engagement littéraire différent mais tout aussi caractéristique. Nous nous sommes en effet efforcée de décrire le fonctionnement de sa pratique de la "glose intégrante ", qui vise à poser un "récit initiatique interculturel », en cohérence avec le positionnement spécifique de l'auteur dans le champ littéraire et politique de la francophonie.

Reste que le contrat didactique ainsi engagé par les trois auteurs est d'un genre particulier. À la différence des « séquences potentiellement acquisitionnelles dans la conversation exolingue » (De Pietro, Matthey \& Py 1988), il n'est pas négociable par le lecteur, contraint de l'accepter pour poursuivre sa lecture, et il faut l'envisager comme une expression possible des «stratégies d'émergence » (Halen 2001) permettant la définition d'un champ littéraire dans le cadre du «système littéraire francophone ». Faisant office de révélateur de ces stratégies d'émergence, l'analyse systématique de ce contrat offre à nos yeux des ressources prometteuses pour les études littéraires francophones.

Pour finir, notons que son examen sera encore précieux à un autre titre, car en même temps que ce contrat permet à l'auteur de se situer dans le champ littéraire francophone, il modifie profondément le tissu du récit lui-même. Les analyses du traitement de l'emprunt lexématique permettront alors de tester l'hypothèse de Gauvin, selon laquelle la note en bas de page glosant l'emprunt, loin d'être marginale par rapport au corps du récit, « intervient » jusque « dans la diégèse » $(2009: 21)$ : pour elle, le traitement de l'emprunt par l'auteur met implicitement en œuvre « une nouvelle poétique romanesque ». Consciente de l'importance décisive de cette piste, nous avons tenté ailleurs (Schwob 2014b) de découvrir quelles "poétique[s] du divers » (Glissant 1996), susceptibles d'élaborer le tissu conjonctif d'un nouveau récit, régissent dans les trois romans du corpus l'insertion de l'emprunt et son traitement. Nous espérons que l'examen du corpus d'ethnonymes mis en œuvre dans le présent article aura contribué à montrer comment les pratiques de la glose par les auteurs hétérolingues peuvent constituer des entrées significatives dans l'étude de leur style, le cas des ethnonymes étant à nos yeux particulièrement révélateur de la pertinence des analyses ethnostylistiques appliquées à ces littératures.

\section{Références bibliographiques}

Amossy, R. (1997). Stéréotypes et clichés : langue, discours et société. Paris : Nathan Université.

Amselle, J.-L. (1985). Ethnies et espace : pour une anthropologie topologique. In J.-L. Amselle \& \& E. M'Bokolo 
Elikia (Dirs.), Au cæur de l'ethnie : ethnie, tribalisme et État en Afrique. Paris : La Découverte, 11-48.

Authier-Revuz, J. (1995). Ces mots qui ne vont pas de soi. Boucles réflexives et non-coüncidences du dire. Paris : Larousse.

Bal, W. ([1983], 1988). Introduction. Genèse et travaux de base. In Équipe IFA (A.E.L.I.A.), D. Racelle-Latin (Coord.), Inventaire des particularités lexicales du français en Afrique noire. Paris : EDICEF/AUPELF, 922.

Baroni, R. (2004). La coopération littéraire : le pacte de lecture des récits configurés par une intrigue. En ligne sur le site de Fabula : L'atelier de théorie littéraire : www.fabula.org/atelier.php

Barry, A. O. (2006). Pour une sémiotique trans-culturelle de l'écriture francophone d'Afrique. In Appropriation de la langue française dans les littératures francophones de l'Afrique subsaharienne, du Maghreb et de l'Océan Indien : Actes des journées scientifiques des réseaux de chercheurs concernant la langue et la littérature . Paris : AUF, 3-14.

Bauer, G. ([1985] 1998). Namenkunde des Deutschen. Bern : Germanistische Lehrbuchsammlung Band 21.

Bazin, J. (1985). À chacun son Bambara. In J.-L. Amselle \& E. M'Bokolo Elikia (Dirs.), Au cour de l'ethnie : ethnie, tribalisme et État en Afrique. Paris : La Découverte, 87-127.

Benzakour, F. (2000). Le français au Maroc. Le problème des doublets : entre dénotation et connotation. In Contacts de langues et identités culturelles : perspectives lexicographiques : Actes des quatrièmes Journées Scientifiques du Réseau "Etude du français en francophonie". Québec : Presses de l'Université Laval ; Collection AS : Actualité Scientifique (AUF), 313-323.

Benzakour, F. (2010). La définition en terre de variation. Le cas du français au Maroc. Publifarum, 11. En ligne : http://publifarum.farum.it/ezine_articles.php?id=115

Bergounioux, G. (2013). Émergence et représentation de la parole intérieure dans la littérature française. Journal of Language and Culture, 8, 1-31. En ligne : http://www.lc.osakafu-u.ac.jp/Lng_Clt/toc.html

Bitjaa Kody, Z. D. (2000). Théorie de l'emprunt à une langue minoritaire : le cas des emprunts du français aux langues africaines. In Contacts de langues et identités culturelles : perspectives lexicographiques : Actes des quatrièmes Journées Scientifiques du Réseau "Etude du français en francophonie". Québec : PUL ; Collection AS : Actualité Scientifique (AUF), 259-268.

Caitucoli, C. (2007). Ahmadou Kourouma et l'appropriation du français : théorie et pratique. Synergies, 2, 5370.

Calvet, L.-J. (2006). Externe, interne, horizontale ou verticale : quelle diversité défendre ? Synergies monde, 1, 57-61.

Cheriguen, F. (1987). Barbaros ou Amazigh. Ethnonymes et histoire politique en Afrique du Nord. Mots, 15, 7-22.

Cheriguen, F. (1994). Anthropo-toponymie et désignation de l'"environnement politique". Mots, 39, 93-98.

Ciavolella, R. (2008). Le pouvoir aux marges. Les Fulaabe et l'État mauritanien. Mémoire de doctorat en anthropologie non publié, Université de Milano Bicocca, Milan ; École des Hautes Études en Sciences sociales, Paris.

Dahech, D. (2013). La nominalisation, un processus multidimensionnel, approche contrastive. In E. Ballardini, R. Pederzoli, S. Reboul-Touré \& G. Treguer-Felten (Éds.), Les facettes de l'évènement, des formes aux signes, MediAzioni 15. En ligne : http://mediazioni.sitlec.unibo.it

De Pietro, J.-F. (1988). Vers une typologie des situations de contacts linguistiques. Langage et Société, 43, 65-89.

De Pietro, J.-F., Matthey, M. \& Py, B. (1988). Acquisition et contrat didactique : les séquences potentiellement acquisitionnelles dans la conversation exolingue. In Actes du III Colloque Régional de Linguistique : Strasbourg 28-29 avril 1988. Strasbourg: Université des Sciences humaines et Université Louis Pasteur, 99-119.

Diop, P. S. (2003). Discours nationaliste et identité ethnique à travers le roman sénégalais. Paris : L'Harmattan.

Diop, P. S. (2010). Glossaire du Roman Sénégalais. Paris : L'Harmattan.

Diouf, M. (2006). Les études postcoloniales à l'épreuve des traditions intellectuelles et des banlieues françaises. Contretemps, 16, 21-34.

Dubois, J., Guespin, L., Giacomo, M., Marcellesi, C., Marcellesi, J.-B. \& Mével, J.-P. ([1973] 1994). Dictionnaire de la linguistique et des sciences du langage. Paris : Larousse.

Durieux, C. (2010). Traduire l'intraduisible : négocier un compromis. Meta : journal des traducteurs / Meta : Translators' journal, 55 (1), 23-30.

Frey, C. (2008a). Regards de locuteurs francophones sur la diversité lexicale en Afrique. Représentations, identités, intercompréhension. In actes du colloque : La langue française dans sa diversité . Québec : Direction des Relations publiques du ministère de la Culture, des Communications et de la Condition féminine, 17-38.

Frey, C. (2008b). Description lexicographique et idéologies : jusqu'où s'engager? In C. Bavoux (Dir.), Le français des dictionnaires. L'autre versant de la lexicographie française (pp. 243-255). Bruxelles : De BoeckDuculot.

Froidevaux, S. (2005). Nommer l'autre. Typologies, ethnonymes et stéréotypes. In E. Deuber Ziegler \& G. Perret 
(Dirs.), Nous autres. Genève : M. E. G., 67-90.

Galisson, R. (1988). Cultures et lexicultures. Pour une approche dictionnairique de la culture partagée. Annexe des Cahiers de linguistique hispanique médiévale, 7, 325-341.

Gauvin, L. (2009). L'écrivain francophone et ses publics. Bruxelles : Académie royale de langue et de littérature françaises de Belgique.

Gleßgen, M.-D. \& Thibault, A. (2005). La «régionalité linguistique » dans la Romania et en français. In M.-D. Gleßgen \& A. Thibault, La Lexicographie différentielle du français et le Dictionnaire des régionalismes de France. Strasbourg : PUS, 281-297.

Glissant, É. Introduction à une poétique du divers. Paris : Gallimard.

Gouvert, X. (2008). Problèmes et méthodes en toponymie française. Essais de linguistique historique sur les noms de lieux du Roannais. Mémoire de doctorat en Langue française non publié, Université de Paris-Sorbonne, Paris.

Gruénais, M.-É. (1986). Quelles ethnies pour quelle anthropologie ? L'Homme, 26 (97-98), 355-364.

Grutman, R. (1996). Des langues qui résonnent. L'hétérolinguisme au XIXe siècle québécois. Montréal : FidesCETUQ.

Guèye, M. (2005). Aminata Sow Fall. Oralité et société dans l'œuvre romanesque. Paris : L'Harmattan.

Guilbert, L. (1975). La créativité lexicale. Paris : Larousse.

Halen, P. (2001). Constructions identitaires et stratégies d'émergence : notes pour une analyse institutionnelle du système littéraire francophone.Études françaises, 2 (37), 13-31.

Hulstijn, J. H. (1994). L'acquisition incidente du lexique en langue étrangère au cours de la lecture : ses avantages et ses limites. Acquisition et interaction en langue étrangère, 3, 77-96.

Jonasson, K. (1994). Le nom propre. Constructions et interprétations. Paris : Duculot.

Lafage S., 2003, Le lexique français de Côte d'Ivoire: appropriation \& créativité, Institut de linguistique française \& CNRS, Nice, 2 vol.

Maalouf, A. (1983). Les Croisades vues par les Arabes. La barbarie franque en Terre sainte. Paris : J.-C. Lattès.

Maingueneau, D. (1993). Le contexte de l'œuvre littéraire. Paris : Dunod.

Manessy, G. (1995). Créoles, pidgins, variétés véhiculaires : Procès et genèse. Paris : CNRS.

Mejri, S. (2012). Les spécificités du français en Tunisie : emprunts autochtones, « géosynonymes » et mots construits. Le français en Afrique, 27, 219-228.

Mendo Zé, G. (2004). Introduction à la problématique ethnostylistique. Langues et Communication, 1 (4), 15-35.

Mendo Zé, G. (2009). Commentaire ethnostylistique d'une fable de La Fontaine : «Le Corbeau et le renard». Langues et communication, 7, 17-43.

Mendo Zé, G. (2010). Cahier d'un retour au pays natal. Aimé Césaire. Approche ethnostylistique. Paris : L'Harmattan.

Mendo Zé, G., Tonye, A. \& Noumssi, G.-M. (2009). S... comme stylistiques. Propositions pour l'ethnostylistique. Paris : L'Harmattan.

Molinié, G. (1994). Le style en sémiostylistique. In G. Molinié \& P. Cahné (Dirs), Qu'est-ce que le style ? Paris : PUF, 201-211.

N'Diaye Corréard, G. \& Schmidt, J (1987-1988). Quelques remarques sur l'étude du français d'Afrique. Bulletin de l'Observatoire du Français Contemporain en Afrique Noire, 7, 137-164.

Ngo Lend, C. E. (2010). La culture africaine au travers de l'énoncé. Une lecture ethnostylistique de Mémoires de Porc-épic d'Alain Mabanckou. Anadiss, 9, 90-145.

Ngo Lend, C. E. \& Fonkua, P. (2010). Hétéroglossie et hétérogénéité littéraires dans la prose romanesque d'Alain Mabanckou. Anadiss, 9, 90-145.

Nicolaï, R. (2000). La traversée de l'empirique. Essai sur la construction des représentations de l'évolution des langues. Paris: Ophrys.

Nicolaï, R. (2001). Linéament. In R. Nicolaï (Éd.), Leçons d'Afrique : Filiations, ruptures et reconstitutions des langues ; un Hommage à Gabriel Manessy. Paris : Peeters, 21-39

Pohl, J. (1985). Le français de Belgique est-il belge ? Présence francophone, 27, 9-19.

Poirier, C. (1995). Les variantes topolectales du lexique français. Proposition de classement à partir d'exemples québécois. In M. Francard et D. Latin (Eds.), Le régionalisme lexical. Louvain-La-Neuve : Duculot, 13-56.

Prince, G. (1973). Introduction à l'étude du narrataire. Poétique, 14, 178-196.

Queffélec, A. (1998). Des migrants en quête d'intégration : les emprunts dans les français d'Afrique. Le Français en Afrique, 12, 245-256.

Raccah, P.-Y. (1990). Signification, sens et connaissance : une approche topique. Cahiers de Linguistique Française, $11,179-198$.

Raccah, P.-Y. (2002). Lexique et Idéologie : les points de vue qui s'expriment avant qu'on ait parlé. In M. Carel 
(Dir.), Les Facettes du dire, Hommages à Oswald Ducrot. Paris : Kimé, 241-268.

Raccah, P.-Y. (2011a). Racines lexicales de l'argumentation : la cristallisation des points de vue dans les mots. Verbum, 32 (1), 119-141.

Raccah, P.-Y. (2011b). Les questions de rhétorique sont-elles des questions sémantiques ? Réflexions sur une théorie de la signification, informées par des études de sémantique contrastive. Revue de sémantique et pragmatique, 29-30, 151-173.

Raccah, P.-Y., Ben Mahfoudh-Hubert, H. \& Chmelick, E. (2011). Les points de vue dans le lexique et dans le dictionnaire. In F. H. Baider, E. Lamprou \& M. Monville-Burston (Eds.), La marque en lexicographie. Limoges : Lambert Lucas, 117-135.

Rastier, F. (1990). La triade sémiotique, le trivium et la sémantique linguistique. Nouveaux Actes Sémiotiques, 9, 553.

Rastier, F. ([1991] 2001). Sémantique et recherches cognitives. Paris : PUF.

Rastier, F. (2003). Le silence de Saussure ou l'ontologie refusée. In S. Bouquet (Éd.), Saussure. Paris : L'Herne, 2531.

Rastier, F. (2006). Sémiotique et sciences de la culture. Une introduction. Texto ! En ligne : http://www.revuetexto.net/Inedits/Rastier/Rastier Post-humanite.html

Rastier, F. (2007). Du réalisme au postulat référentiel. Texto ! En ligne : http://www.revuetexto.net/Inedits/Inedits.html

Robert, P., Rey-Debove, J. \& Rey, A. (2014). Le Petit Robert : Dictionnaire alphabétique \& analogique de la langue française. Paris : Société du Nouveau Littré.

Schwob, D. (2014a, à paraître). Être enseignant-passeur de littératures francophones dans le secondaire français : vers quelles compétences interculturelles pour les classes plurilingues ? In M. Rispail. \& J.-F. de Pietro (Coords.), L'enseignement du français à l'heure du plurilinguisme. Pour une didactique contextualisée. Namur : Presses Universitaires de Namur - AIRDF.

Schwob, D. (2014b, à paraître). Personnages en quête de référence(s) dans trois romans hétérolingues en langue française : pour une poétique comparée de la glose. Fabula-LHT, 12.

Schwob, D. (2014, juin). Un narrateur, des narrataires, et beaucoup d'emprunts... Trois romans hétérolingues à lire entre acquisition incidente et contrat didactique. Communication présentée au $3^{\mathrm{e}}$ colloque pluridisciplinaire autour de l'étude du langage du CEDIL, Grenoble.

Steuckardt, A. (2006, mars). Du discours au lexique : la glose. Communication présentée au séminaire ATLIF, Nancy. En ligne : http://web.atilf.fr/img/pdf/seminaires

Steuckardt, A. (2009). Les mots dans l'histoire. Représentations de la langue, rhétorique et stylistique des discours, changement lexical. Mémoire de H.D.R. en Sciences du langage non publié, Université de Provence, Aixen-Provence.

Stratford, M. (2008). Au tour de Babel ! Les défis multiples du plurilinguisme. Meta : journal des traducteurs / Meta : Translators' journal, 53 (3), 457-470.

Sultani, J.C. \& Milelli J.P. (2010). Dictionnaire Français-Libanais. Versailles : Éditions Milelli.

Thibault, A. (2006). Glossairistique et littérature francophone. Revue de linguistique romane, 277-78, 143-179.

Thibault, A. (2012). Francophonie et variété des français. Cours de Licence de Langue Française. Semaine 7 : Le français dans les Antilles. Document non publié, Université Paris IV-Sorbonne. En ligne : http://andre.thibault.pagesperso-orange.fr

Vaxelaire, J.-L. (2005). Les Noms propres : une analyse lexicologique et historique. Paris : Champion.

1 Certains les appellent « géosynonymes » (Mejri 2012).

2 L'adaptation de cette méthode à l'analyse des littératures hétérolingues et sa valeur heuristique pour l'établissement de catégories d'analyse littéraires adaptées à ce corpus sont exposées en détail dans Schwob 2014b.

3 L'étude du traitement des interjections dans le roman de Sow Fall est menée avec précision dans Schwob 2014b.

4 Bazin, J. (1985). À chacun son Bambara. In J.-L. Amselle \& E. M'Bokolo Elikia (Dirs.), Au coeur de l'ethnie : ethnie, tribalisme et État en Afrique (pp. 87-127). Paris : La Découverte.

5 Nous ajoutons la nuance.

6 Tout ceci nous ramène toujours à la problématique du lien entre lexique et connaissances partagées. Celle-ci est évoquée notamment par N'Diaye Corréard \& Schmidt (1987-1988 : 141-142) lorsqu'ils 
décrivent, en lien avec la question de l'intercompréhension, l'opposition, au sein du lexique francophone, entre une «zone de consensus » regroupant des lexies communes à la totalité du monde francophone, et une « zone de divergence » dans laquelle les lexies ou leur usage varient, ainsi que des «zones de consensus partielles » au sein de la zone de divergence -, cette dernière notion étant en cohérence notamment avec l'hypothèse sémantactique de Manessy.

7 Pour un aperçu de l'histoire des littératures hétérolingues et monolingues et notamment des représentations sur celles-ci, voir Stratford (2008).

8 L'analyse de ce pacte comme contrat didactique sera menée plus en détail dans une communication intitulée «Un narrateur, des narrataires, et beaucoup d'emprunts... Trois romans hétérolingues à lire entre acquisition incidente et contrat didactique » (Schwob 2014c).

9 Sans pouvoir développer pleinement les enjeux de ce pacte ainsi envisagé, nous esquisserons rapidement ici quelques pistes d'analyse, en renvoyant pour une réflexion plus poussée sur ce sujet à Schwob (2014b).

10 Pour une analyse approfondie du traitement spécifique des emprunts dans Allah n'est pas obligé, traitement que nous qualifions de polyréférentiel, voir Schwob (2014b). 